Ref uel i ng for St eady- St at e PI asma by Repet i t i ve Pel I et I nj ecti on

\begin{tabular}{|l|l|}
\hline $\begin{array}{l}\text { jour nal or } \\
\text { publ i cat i on } \mathrm{titl} \text { e }\end{array}$ & Pl asma Sci ence and Technol ogy \\
\hline vol une & Vol . 6 \\
\hline page range & pp. 2275- 2280 \\
\hline year & 2003 10 - 01 \\
\hline URL & ht t p: //hdl . handl e. net /10655/1311 \\
\hline
\end{tabular}




\title{
Refueling for Steady-State Plasma by Repetitive Pellet Injection in Large Helical Device
}

\author{
H.Yamada ${ }^{\text {a }}$, R.Sakamoto ${ }^{\text {a }}$, I.Viniar ${ }^{\text {b }}$, M.Goto ${ }^{\text {a }}$, K.Kikuchi ${ }^{\text {c }}$, A.Lukin ${ }^{\text {b }}$, \\ S.Masuzaki ${ }^{\text {a }}$, J.Miyazawa ${ }^{\text {a }}$, S.Morita ${ }^{\mathrm{a}}$, Y.Oda ${ }^{\mathrm{c}}$, S.Sudo ${ }^{\mathrm{a}}$, K.Tanaka ${ }^{\mathrm{a}}$, \\ LHD Experimental Group \\ a National Institute for Fusion Science, Toki, Japan \\ ${ }^{b}$ PELIN Laboratory, Ltd.,Moscow, Russia \\ ${ }^{c}$ Mitsubishi Heavy Industries, Ltd., Kobe, Japan \\ hyamada@lhd.nifs.ac.jp
}

Keywords : Cryo-cooler, Screw extruder, Solid hydrogen

\begin{abstract}
A repetitive pellet injector has been developed for investigation of refueling issues towards the steady-state operation in Large Helical Device (LHD). Continuous operation of more than 10000 pellet launches at $10 \mathrm{~Hz}$ has been demonstrated. The maximum repeating rate is $11 \mathrm{~Hz}$. No technical constraint for longer operation has been found. The reliability of pellet launch has exceeded 99.9\%. The initial application to the NBI heated plasmas has been successful in the last experimental campaign of LHD. Although the pulse length is limited by the operational constraint of NBI, the plasma with density of $8 \times 10^{19} \mathrm{~m}^{-3}$ has been sustained for $2 \mathrm{~s}$ by the pellet injection at $10 \mathrm{~Hz}$. A prospect for the future experiment is discussed on basis of the initial result.
\end{abstract}

\section{Introduction}

Fueling is a primary element of particle control in a fusion research. High temperature plasmas with large dimension in recent large-scale experiments have posed an issue of inevitability of gas-puffing since a thick and hot scrape-off layer prevents the penetration of neutrals and consequently the fueling efficiency is degraded seriously. This problem is highlighted in the long pulse operation towards the steady-state [1]. Hydrogen ice pellet injection has a potential to resolve these problems; however, a full-scale plasma experiments in steady-state by pellet injection have not be done yet.

The refueling issues have been intensively investigated in Large Helical Device (LHD) which is a largest stellarator in the world. The intrinsic physical advantage of a heliotron concept and employment of a superconducting magnet system provide capability of steady-state operation of LHD [2]. Since the fueling efficiency of gas puffing is limited around $10 \%$ [3], alternative efficient fueling schemes are required for steady-state high-performance plasma experiments. The pellet injection experiments conducted to date have used the pellet injector which employs a conventional mechanism of a pipe gun and the GM refrigerator instead of liquid helium. This pellet injector has successfully eliminated troublesome factors [4]. Consequently reliability of fueling pellet injection has exceeded $99 \%$ and the pellet injection routinely has served plasma experiments as well as gas-puff[5]. In this injector, however, the number of available pellets is limited to 10 during one plasma discharge since ice pellets are formed in a barrel. A long pulse operation of high temperature plasmas has been already demonstrated for more than 2 minutes [6]. The prolonged discharge exceeding $10^{4}$ seconds is in plan. Therefore a new repetitive fueling pellet injector is required to enable the experimental approach towards steady-state operation in LHD. The new injector adopts the screw extrusion concept, proposed by Mitsubishi Heavy Industry [7] and 
developed by PELIN Laboratory [8,9]. Demonstration of basic performance and an initial plasma experiment are reported in this article.

\section{Specification and Technical Elements}

The specification has been determined from the experimental data using the presently available pellet injector. The particle contained in one pellet is $3-6 \times 10^{20}$ to fit the objective of a new repetitive injector, which leads to the size with $2-2.5 \mathrm{~mm}$ in diameter. One pellet injection results in a moderate density increase of around $1-2 \times 10^{19} \mathrm{~m}^{-3}$. The maximum repetition rate is set at larger than $10 \mathrm{~Hz}$ which gives the interval shorter than a typical particle confinement time. Although large launching velocity is favourable for deep penetration, controllability of the velocity is required to secure the transfer of intact pellets in the curved guide tube [10] which provides a large flexibility of injection locations [6]. Therefore the the pellet velocity should be controlled between 100 and $700 \mathrm{~m} / \mathrm{s}$. The minimum capability of operational duration is $1000 \mathrm{~s}$ to fit the present goal of the long pulse operation in LHD, however, in principle, there should not be a technical limitation of the operational duration. A solidification system of hydrogen using a screw-extruder combined with the cutting mechanism is adopted. Also reliability of the system should be highly considered because this pellet injector has to work routinely as a primary experimental tool and the large scale superconducting device does not allow an easy accessibility during machine operation. From this point of view, a simple acceleration by a propellant gas and refrigeration by a GM cryo-cooler are adopted. Figure 1 is a schematic view of the repetitive pellet injection system.

\section{Demonstration of Performance of Major Engineering Elements}

\section{3-1 Cooling Capability}

Refrigeration is done by two GM-type cryo-coolers (20 W at $8 \mathrm{~K}$ in total). Any cooling media like liquid helium are not used in the system and the cryo-system is completely closed. This concept has realized a high reliability and an easy maintenance. Also the control of temperature is facilitated and the turn-around time of an operation and a standstill are shortened. Figure 2 shows the cooling-down process of the temperature at the bottom of extruder. The pellet injection is available 4 hours after the start of cooling down from the room temperature. Also it takes only 1 hour to recover the cryogenic temperature sufficient for the pellet injection from the 6-hour pause of cooling. The temperature at the extruding position is as low as $6 \mathrm{~K}$ without pellet formation while the typical operational temperature is $10-11 \mathrm{~K}$. The temperature is controlled by $30 \mathrm{~W}$ electric heater. Figure 3 shows the temperature rise during the pellet injection.

\section{3-2 Solid hydrogen extrusion}

Hydrogen is solidified continuously in the cryogenic screw extruder with the maximum rate of $15 \mathrm{mg} / \mathrm{s}$. There is no limitation of the operational duration in a technical principle. The solidified hydrogen rod is extruded with a diameter of $2.0 \mathrm{~mm}$ or $2.5 \mathrm{~mm}$ and consequently the extrusion of $55 \mathrm{~mm} / \mathrm{s}$ or $35 \mathrm{~mm} / \mathrm{s}$ is provided, respectively. The continuous extrusion of solid hydrogen has been demonstrated for $1000 \mathrm{~s}$ with the rate of $35 \mathrm{~mm} / \mathrm{s}$ and the diameter of 2.5 $\mathrm{mm}$. The size and its deviation of launched pellets are investigated to check the quality of solid hydrogen as a function of formation temperature. When the temperature at the extruding position is less than $11.3 \mathrm{~K}$, the size and its deviation is maintained stable. When the temperature increases above $12 \mathrm{~K}$, the deviation of the size becomes large, which suggests the deterioration of quality of pellets. Since the cooling capability is sufficient for the heat load during solidification and extrusion and the increase of temperature is limited to much less than $1 \mathrm{~K}$ (see Fig.3) even at the maximum extrusion speed, the favourable operational temperature can be kept for steady-state operation. 


\section{3-3 Cut and launch}

The extruded hydrogen rod is cut by the cutter sliding along the barrel and launched by a propellant helium gas. Both actions are driven by two synchronizing magnetic solenoids. Figure 4 shows the amount of consumed propellant gas per one pellet launch and the velocity of launched pellets as a function the pressure of propellant gas. These characteristics do not change for the repetitive frequency between 1 to $10 \mathrm{~Hz}$. Since a simple mechanism of a gas gun is employed, the amount of consumed propellant gas is a critical issue, in particular, for steady-state operation. The amount of gas is suppressed to less than $1.5 \mathrm{Pam}^{3}$ per one pellet launch. The velocity declines above $3 \mathrm{MPa}$ due to deterioration of the opening motion against the pressure by the solenoid while the velocity of the pellet can be controlled from $150 \mathrm{~m} / \mathrm{s}$ to $550 \mathrm{~m} / \mathrm{s}$. The probability is also simultaneously evaluated from the number of intact pellet measured by the shadow graph for 10000 launches at $10 \mathrm{~Hz}$. Below $3 \mathrm{MPa}$, the probability is very close to $100 \%$. Here it should be pointed out that both probability and the range of velocity could be improved in the case with higher pressure than 3MPa since the mechanical motion of the solenoid can be also controlled by the applied voltage.

\section{3-4 Differential pumping}

The flow of propellant gas into the plasma vacuum vessel should be suppressed not to affect plasma discharges. The maximum gas flow is $17 \mathrm{Pam}^{3} / \mathrm{s}$. Three expansion chambers with pumps are employed between the chamber for the pellet launch and the plasma vacuum vessel. The first chamber has the volume of $70 \mathrm{l}$ and two-stage pumping unit of a helical grooved pump (390l/s) and a roots pump $\left(1800 \mathrm{~m}^{3} / \mathrm{h}\right)$. The second and third chambers have the volumes of $70 \mathrm{l}$ and 15l, and a helical grooved pump (390l/s) and a turbo drag pump (400l/s), respectively. Figure 5 shows the simulation result of the evolution of vacuum in each chamber in $10 \mathrm{~Hz}$ operation. Vacuum condition saturates within 1s. Vacuum in the last 3rd chamber which is connected to the LHD plasma vacuum vessel is maintained steadily to be less than $10^{-6} \mathrm{~Pa}$. This good condition has been confirmed in the experiment.

\section{3-5 Engineering commissioning}

A successful technical integration of major elements has led to the completion of a repetitive pellet injector for LHD. 10000 continuous launches at $10 \mathrm{~Hz}$ have been demonstrated. The quality of the pellet is verified by a shadow graph and reliability of intact pellet launch is more than $99.9 \%$. Any troubles have not taken place in this long operation, which indicates no technical constraints for real steady-state operation. The pellet mass can be also reduced by slowdown of extruding speed from the maximum mass of $6 \times 10^{20}$ hydrogenic particles.

\section{Initial Experimental Results}

Repetitive pellet injection has been applied to refuelling to NBI heated plasmas in LHD. Figure 6 is waveforms of a typical plasma discharge with 50 pellet injections for $5 \mathrm{~s}$. Quasi-steady state with density as high as $8 \times 10^{19} \mathrm{~m}^{-3}$ has been maintained from $3.6 \mathrm{~s}$ to $5.6 \mathrm{~s}$. Heating power of NBI in this phase is $4 \mathrm{MW}$. The pellets penetrate to $\rho=0.6$ a (see Fig.6(a). Note that the full minor radius along the pellet path is $0.8 \mathrm{~m}$ ), however, perturbation to the central temperature is very small. Although the line averaged density and the central electron and ion temperatures are kept constant, the stored energy gradually declines, which suggests degradation of confinement in the peripheral region. The envelopes of $\mathrm{H} \alpha$ signal as well as the particle flux on the divertor plate increase monotonically. Simultaneously the neutral gas pressure increases. When neutral pressure exceeds $2 \times 10^{-3} \mathrm{~Pa}$, density stops increasing and degradation of confinement starts. These observations suggest that excess neutrals from recycling degrade plasma performance. 
Figure 7 shows a comparison of density ramp-up discharges with different refuelling methods. It has been widely known that energy confinement time in net-current-free plasmas can be expressed by the ISS95 scaling [11].

$\tau_{E}^{I S S 95}=0.079 a^{2.21} R^{0.65} P^{-0.59} \bar{n}_{e}^{0.51} B^{0.83} \boldsymbol{t}_{2 / 3}^{0.4}$

where the heating power $P$ and $\bar{n}_{e}$ have units of MW and $10^{19} \mathrm{~m}^{-3}$, respectively. $\boldsymbol{t}_{2 / 3}$ is the rotational transform at two thirds radius. This scaling indicates that the stored energy evolves with the density as $\bar{n}_{e}^{0.49}$. Similar significant positive dependence on density has been confirmed in the LHD experiment [12]. However, in the case with refuelling by gas puff, pronounced saturation and/or degradation of confinement is observed in the high density regime (see a thin curve in Fig.7). This degraded state can be maintained for 10s. In the case with refuelling by several large pellets with a conventional pipe gun [4], the envelope of the trajectory follows the prediction of ISS95 and a transient confinement improvement is observed after pellet injection (see a dotted curve in Fig.7). The trajectory in the case with repetitive pellet injection seems to show better performance than other two methods which can be attributed to better fueling efficiency of repetitive pellet injection than gas puff and smaller damage on plasma confinement than larger pellets with longer interval.

\section{Discussions and Conclusions}

A repetitive pellet injector has been developed for investigation of refueling issues towards the steady-state operation in LHD. Continuous operation of more than 10000 pellet launches at $10 \mathrm{~Hz}$ has been demonstrated. The maximum repeating rate is $11 \mathrm{~Hz}$ and no technical constraint has been found for longer operation. The reliability of pellet launch has exceeded 99.9\%. The initial application to the NBI heated plasmas has been done in the last experimental campaign of LHD. $10 \mathrm{~Hz}$ repetitive pellet injection for $6 \mathrm{~s}$ has been successfully performed. Quasi-steady state operation for $2 \mathrm{~s}$ has been achieved with major plasma parameters of $\bar{n}_{e}$ of $0.8 \times 10^{20} \mathrm{~m}^{-3}, \mathrm{~T}_{\mathrm{i}}(0)$ of $1.3 \mathrm{keV}, \mathrm{T}_{\mathrm{e}}(0)$ of $1.0 \mathrm{keV}$ with $4 \mathrm{MW}$ NBI heating. Repetitive pellet fueling shows improved energy confinement compared with massive gas puff at high density region. However, gradual confinement degradation is observed with keeping high density. In this phase, an increase of recycling particles is observed.

Repetitive pellet injection has certainly suppressed excess neutrals in high density operation compared with gas puff. However, fueling efficiency less than $100 \%$ and lost particles from the confined plasma result in excess neutrals around the plasma sooner or later. Therefore pumping capability compatible with the refuelling scheme is requisite for high-density steady state operation. The maximum fuelled particle by repetitive pellets equivalent to $10 \mathrm{Pam}^{3} / \mathrm{s}$ of hydrogen gas. A new facility of Local Island Divertor(LID) has been recently available [13]. The pumping capability of this innovative divertor is $42 \mathrm{~m}^{3} / \mathrm{s}$ and the overall pumping efficiency of more than $30 \%$ is anticipated. The pressure in the divertor chamber of $0.6 \mathrm{~Pa}$, which is still a challenging value but in our scope, or good particle confinement mitigating the repetition rate is required to control the excess neutrals. Combined experiment of the LID and repetitive pellet injection is planned in the nearest future.

\section{Acknowledgement}

The authors are grateful to Dr.S.Combs, Dr.P.Fisher, Dr.M.Gouge and Dr. S.Milora of Oak Ridge National Laboratory for their contributions and communications to the development of pellet injection in LHD. Tremendous effort for LHD operation by the device engineering group of LHD is also gratefully acknowledged. 


\section{References}

[1] L.R.Baylor, T.V.George, M.Gouge et al., Fusion Technol. 34 (1998) 86.

[2] A.Iiyoshi, A.Komori, A.Ejiri et al., Nucl. Fusion 11Y (1999) 1659.

[3] J.Miyazawa, S.Masuzaki, H.Yamada et al., J. Nucl. Materials 313-316 (2003) 534.

[4] H.Yamada, R.Sakamoto, Y.Oda et al., Fusion Eng. Des. 49-50 (2000) 915.

[5] R.Sakamoto, H.Yamada, K.Tanaka et al., Nucl. Fusion 41 (2001) 381.

[6] H.Yamada, A.Komori, N.Ohyabu et al., Plasma Phys. Control. Fusion 43 (2002) A55.

[7] Y.Oda, M.Onozuka, S.Kasai, K.Hasegawa, Proc. 18th Symp. on Fusion Technology (1994) North Holland, Vol.1, p.661.

[8] I.Viniar, S.Skoblikov, Instrum. Exp. Tech. 5 (2000) 722.

[9] I.Viniar, S.Sudo, A.Geraud et al., Fusion Eng. Des. 58-59 (2001) 295.

[10] S.K.Combs, L.R.Baylor, P.W.Fisher et al., Fusion Eng. Des. 58-59 (2001) 343.

[11] U.Stroth, M.Murakami, H.Yamada et al., Nucl. Fusion 36 (1996) 1063.

[12] H.Yamada, K.Y.Watanabe, S.Sakakibara et al., Phys. Rev. Lett. 84 (2000) 1216.

[13] A.Komori, N.Ohyabu, A.C.England et al., Fusion Eng. Des. 39-40 (1998) 241.

\section{Figure Captions}

Fig.1 Schematic view of the repetitive pellet injection system. The vacuum vessel and magnetic flux surfaces of LHD are illustrated on the left hand side. The pellet injection system has 3 expansion chambers.

Fig.2 A cooling curve of the extruder head from room temperature.

Fig.3 Temperature rise at the extruding position during the repetitive pellet injection. A solid curve is the case with $10 \mathrm{~Hz}$ corresponding to the solidification speed of $15 \mathrm{mg} / \mathrm{s}$. A dotted curve: two thirds of the maximum. A thin solid line : half the maximum.

Fig.4 Consumed propellant gas per one pellet launch and velocity of a pellet as a function of the pressure of a propellant gas.

Fig.5 Calculated increase of vacuum pressure in expansion chambers during a repetitive pellet injection.

Fig.6 Waveforms of NBI heated plasmas refuelled by a repetitive pellet injection. (a) Penetration depth $\lambda$. (b) Stored energy $W_{\mathrm{p}}$ and heating power of NBI $\mathrm{P}_{\mathrm{NBI}}$. (c) Central

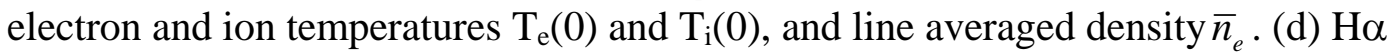
signal and particle flux on to the divertor plate $\Gamma_{\text {div }}$ (e) Neutral pressure $\mathrm{p}_{0}$ and total radiation power $\mathrm{P}_{\text {rad. }}$.

Fig.7 Trajectory of evolution of stored energy of discharges with density ramp-up as function of line averaged density. A thin solid curve is the case with normal gas puff. A dotted curve is the case with larger $\left(1 \times 10^{21}\right.$ particles) pellet injection from a conventional pipe gun. A fat solid curve is the case with repetitive pellet injection shown in Fig.6. A dashed curve is a prediction from the ISS95 scaling as a reference. 


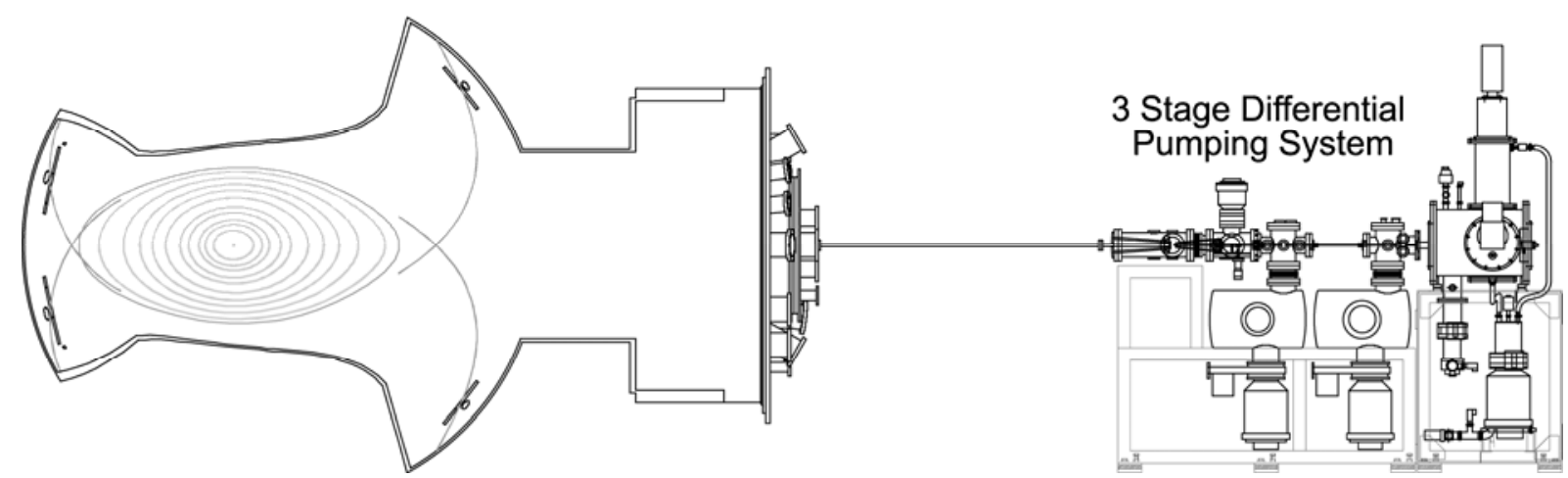

Fig.1

"Refueling for Steady-State Plasma by Repetitive Pellet Injection in Large Helical Device” by H.Yamada et al. (AFPA2003, O-25)

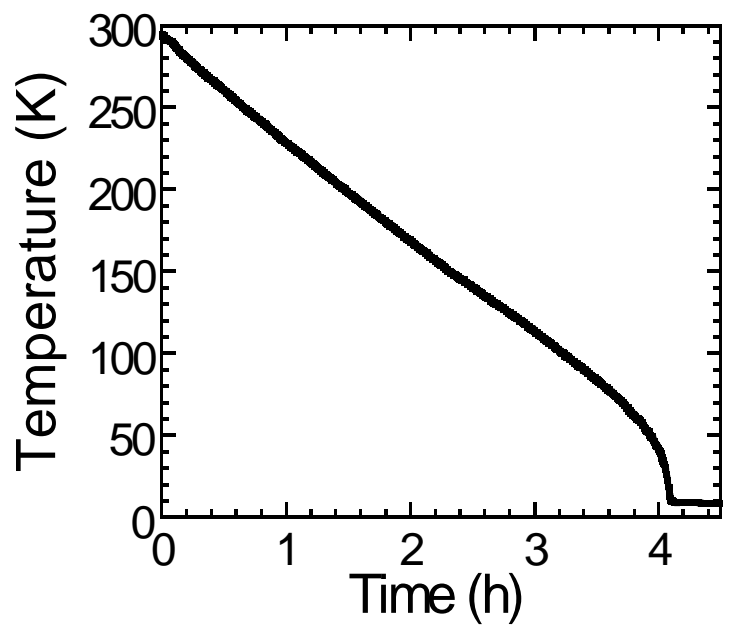

Fig.2

"Refueling for Steady-State Plasma by Repetitive Pellet Injection in Large Helical Device” by H.Yamada et al. (AFPA2003, O-25) 


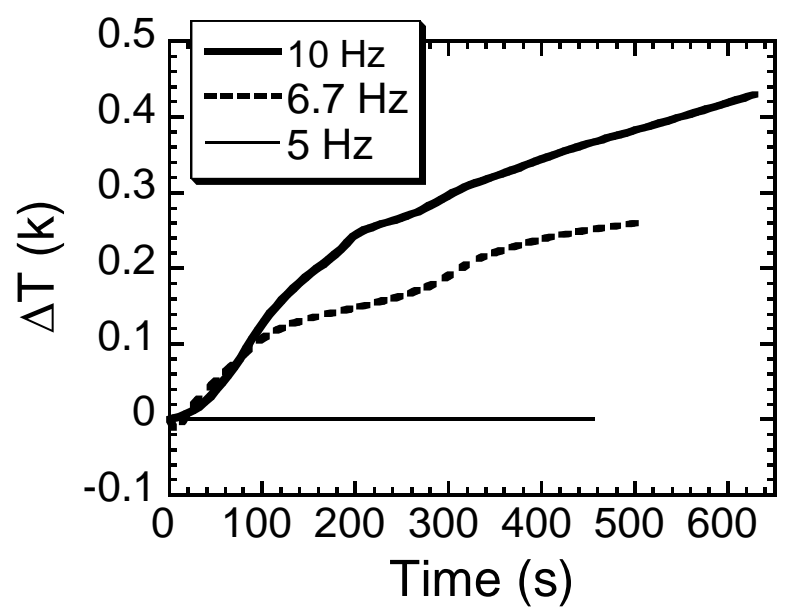

Fig.3

"Refueling for Steady-State Plasma by Repetitive Pellet Injection in Large Helical Device” by H.Yamada et al. (AFPA2003, O-25)

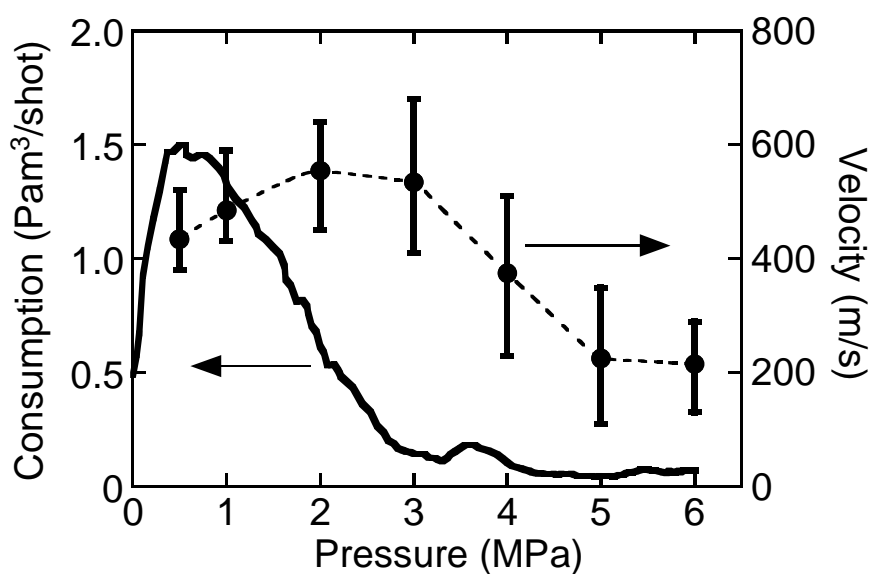

Fig.4

"Refueling for Steady-State Plasma by Repetitive Pellet Injection in Large Helical Device" by H.Yamada et al. (AFPA2003, O-25) 


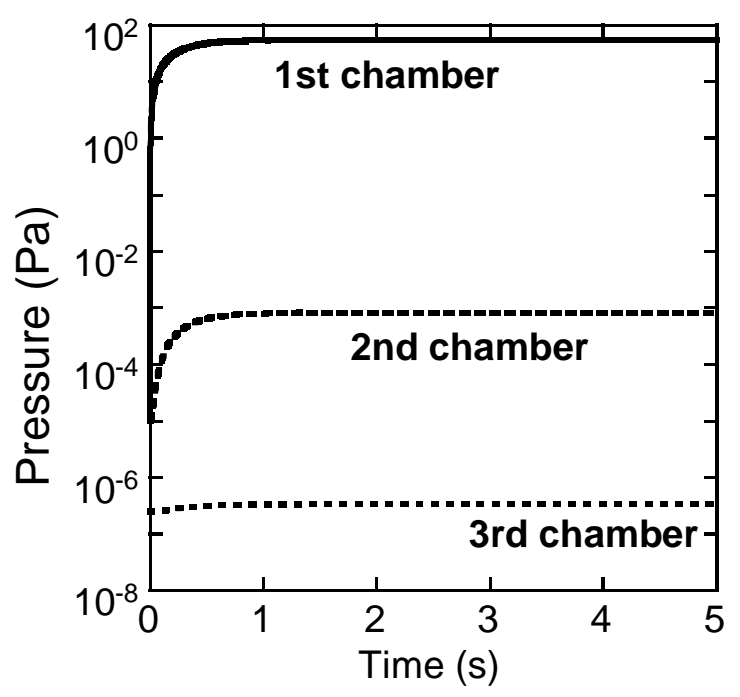

Fig.5

"Refueling for Steady-State Plasma by Repetitive Pellet Injection in Large Helical Device” by H.Yamada et al. (AFPA2003, O-25)

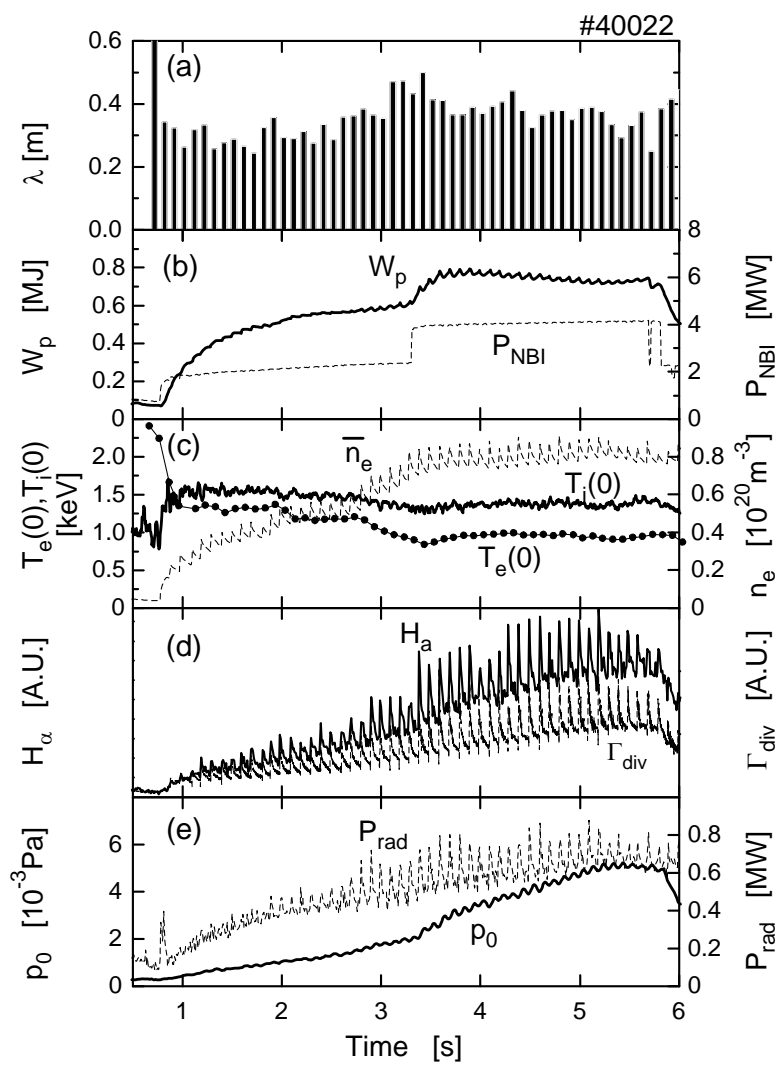

Fig.6

"Refueling for Steady-State Plasma by Repetitive Pellet Injection in Large Helical Device” by H.Yamada et al. (AFPA2003, O-25) 


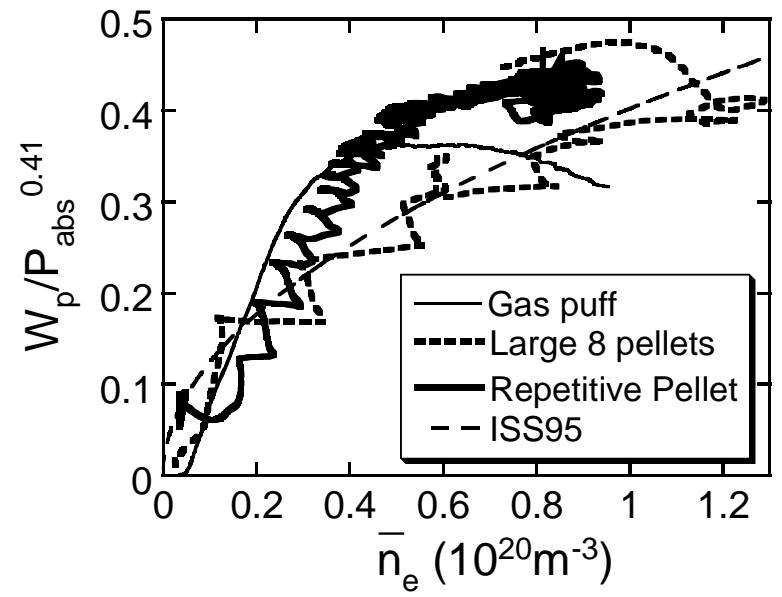

Fig.7

"Refueling for Steady-State Plasma by Repetitive Pellet Injection in Large Helical Device” by H.Yamada et al. (AFPA2003, O-25) 